\title{
Principles of esophageal cancer surgery, including surgical approaches and optimal node dissection (2- vs. 3-field)
}

\author{
Philippe Nafteux, Lieven Depypere, Hans Van Veer, Willy Coosemans, Toni Lerut \\ Department of Thoracic Surgery, University Hospital Leuven, Belgium \\ Correspondence to: Toni Lerut. Department of Thoracic Surgery, University Hospital Leuven, Belgium. Email: toni.lerut@med.kuleuven.be.
}

\begin{abstract}
Surgery for esophageal carcinoma and carcinoma of the gastro-esophageal junction (GEJ) is considered as one of the most complex and challenging interventions on the digestive tract. This is due to the intimate relations with vital structures in the chest and the tendency of early lymphatic dissemination via a dense and complex submucosal network. This review article discusses the different aspects of surgical access routes in the light of the ever-evolving techniques, in particular the minimally invasive esophagectomy (MIE). The aspects of surgical approach are inextricably linked to the still ongoing debate on extent of lymphadenectomy, a debate that is obtaining a new dimension in view of the widely applied neoadjuvant therapy protocols as well as in view of the increasing importance of quality of life aspects after surgery. Finally, the authors provide a practical and patient tailored approach as applied in their center.
\end{abstract}

Keywords: Esophageal cancer; lymph node dissection; transthoracic; transhiatal; minimally invasive esophagectomy (MIE)

Submitted Dec 01, 2016. Accepted for publication Feb 08, 2017.

doi: 10.21037/acs.2017.03.04

View this article at: http://dx.doi.org/10.21037/acs.2017.03.04

\section{Introduction}

The surgical treatment strategy of esophageal carcinoma is complex and the long term outcome of surgical therapy is often disappointing. A malignancy arising from the esophagus may easily invade these adjacent organs, which makes the tumor surgically non-resectable. Additionally, lymphatic dissemination is an early event and has a negative influence on survival. Lymph node metastases are found in less than $5 \%$ of intramucosal tumors but in as much as $30-40 \%$ of submucosal tumors (1). Furthermore, the esophageal wall is characterized by an extensive submucosal lymphatic plexus, which supplies a drainage route for early dissemination and gives rise to skip metastases (i.e., lymph nodes adjacent to the primary tumor are not affected, but more distant-located lymph nodes contain metastases) (2).

As a result transmural tumors are showing lymph node involvement in over $80 \%$ and the number of involved nodes increases with increasing volume of the tumor.

Also adding to the complexity are the tumors of the gastro-esophageal junction (GEJ) that are classified by some as gastric cancer and by some as esophageal cancer.

This explains the ongoing controversy as to which strategy to follow when it comes to surgical approach, surgical techniques and extent of lymphadenectomy for cancers of the esophagus and GEJ.

\section{Principles of surgical treatment}

Surgical strategies for esophageal cancer are based on optimizing oncological results and further limiting complications/mortality so as not to jeopardize the outcomes and quality of life after such extensive undertakings.

\section{Diversity of approaches}

For several decades, the optimal surgical technique for the potentially curative treatment of patients with esophageal cancer has been a matter of debate. The limited transhiatal esophagectomy (THE) was developed in an attempt to mainly minimizing postoperative morbidity/mortality by avoiding a formal thoracotomy but limiting the extent 
of lymph node dissection achievable (3). On the other hand, the transthoracic approach (TTE) with two-field lymphadenectomy (posterior mediastinum, upper abdomen) was introduced as to improve completeness of the resection and to increase locoregional tumor control (4-6). It is widely accepted that extensive lymphadenectomy provides the benefit of a more accurate staging, but its effect on improvement of survival, especially in an era of neoadjuvant treatment followed by surgery is still a matter of debate.

The early-stage lymphatic dissemination as well as completeness of tumoral resection $\left(\mathrm{R}_{0}\right)$ poses challenges for radical surgical treatment and is still a matter of debate and is consequently affecting the choices of surgical strategy and access route.

Indeed in esophageal cancer, lymphatic dissemination occurs early. It has been shown that $20-40 \%$ of all early submucosal (T1b) esophageal tumors have already disseminated to regional lymph nodes $(7,8)$. Therefore, endoscopic treatment, commonly referred to as endoscopic mucosal resection (EMR) is generally reserved for patients with mucosal (T1a) disease (9). Furthermore, the pattern of lymphatic dissemination is unpredictable with skip metastases at more distant sites while lymph nodes in the direct vicinity of the primary tumor are negative (10). Therefore, extended lymphadenectomy, as performed during transthoracic en bloc esophagectomy, theoretically increases the chance of radical removal of all positive lymph nodes claiming to improve regional tumoral control and long-term survival.

In the large retrospective study of the Worldwide Esophageal Cancer Collaboration Group (WECC) on 3,572 patients that underwent $\mathrm{R} 0$ resection $(60 \%$ adenocarcinoma, $40 \%$ squamous cell carcinoma), it was shown that a high total number of resected lymph nodes is an independent prognosticator of improved survival after primary surgery for esophageal or GEJ cancers (11). Furthermore, it was shown in a study by Peyre et al. that the optimal threshold for this survival benefit was removal of at least 23 nodes, and the operation most likely to achieve this number was the en bloc resection (12). These findings are clearly arguing in favor of maximizing the extent of lymphadenectomy.

In contrast, other studies and meta-analysis have questioned these conclusions (13-15).

The THE with limited lymphadenectomy and the transthoracic esophagectomy (TTE) with extended lymphadenectomy have been compared in the prospective randomized controlled HIVEX trial on adenocarcinoma of the esophagus and GEJ and which remains until today the only prospective trial (16). There was no difference in postoperative mortality nor in overall oncologic results, between both groups $(\mathrm{P}=0.45)$, but intraoperative blood loss and short term outcome were significantly better in the THE arm. Nevertheless, in a subsequent subgroup analysis of patients with true esophageal (distal 1/3 or Siewert type 1) cancer, a better long term survival was achieved in the TTE arm, in particular in those patients with a limited number of positive nodes $(\mathrm{P}=0.02)(16)$.

In a recent meta-analysis (14) by Boshier et al., THE was again shown to have improved short-term outcome without jeopardizing long-term oncologic outcomes. Shorter operative times $\mathrm{P}<0.001, \mathrm{CI}: 40-129)$, shortened hospital stay $(\mathrm{P}<0.01, \mathrm{CI}: 1-7)$ with lower respiratory complication rates (OR 1.37, 95\% CI: 1.05-1.79, $\mathrm{P}=0.02$ ) and lower mortality (OR 1.48, 95\% CI: $1.20-1.83, \mathrm{P}<0.001$ ) was seen in the THE group versus TTE. On the other hand, lymph node retrieval was higher in the TTE group with a mean difference of 8 nodes (95\% CI: $1-14$ ), $\mathrm{p}=0.02$ ) and furthermore THE experienced more anastomotic leaks and more recurrent nerve palsies. Moreover the authors warned that these results should be analyzed with caution as more advanced tumors might have been treated preferentially with TTE which could have compromised the potential survival benefit. Indeed, a large volume, multicentric study dealing with more than 17,000 patients failed to confirm the advantage of THE in short term outcomes (17).

Furthermore, the recent interest in ERAS protocols may further decrease the complications rate and improve short term outcomes, especially after transthoracic oesophagectomy narrowing the gap with THE.

All together these considerations on extensiveness of resection and extent of lymphadenectomy seem to favor a TTE as opposed to the transhiatal approach (THE) for which the rationale is merely based on an effort to decrease perioperative morbidity and possibly postoperative mortality.

Minimally invasive esophagectomy (MIE) has the potential to limit surgical trauma and so improves shortterm outcomes by limiting postoperative morbidity and mortality while preserving the thoroughness of the resection and the extent of lymph node dissection. This has been shown in several meta-analysis (18-20) and a prospective randomized controlled trial (21).

In the TIME trial (Dutch study) (21), transthoracic MIE was compared to TTE in 115 patients with resectable esophageal and GEJ cancer. For a comparable number of lymph nodes resected and the same oncologic outcomes, 
MIE was shown to have a lower pulmonary infection rate (RR 0.35, 95\% CI: 0.16-0.78, P=0.005). However, main points of criticism were the limited number of patients included, the limited follow up and the subjectivity of the primary endpoint.

The introduction of videoscopic techniques has over time generated, beside the total MIE, a number of variations, commonly referred to as hybrid MIE. They consist in combinations of partial open, partial videoscopic approaches (22).

Recently, the results of the French MIRO trial have been presented (not yet published as a full paper) (23). This prospective randomized controlled trial on 207 patients compared TTE to hybrid MIE (thoracotomy, laparoscopy). Both postoperative morbidity (OR 0.31, 95\% CI: 0.18-0.55, $\mathrm{P}=0.0001)$ and pulmonary complications $(30.1 \%$ vs. $17.7 \%$, $\mathrm{P}=0.037$ ) were lower in the hybrid group.

These results have led to an increased use of MIE techniques in esophageal and GEJ cancer patients although oncologic long term results are still lacking.

\section{Extent of lymph node dissection: an ongoing debate}

According to the definitions accepted during the consensus meeting of the International Society for the Diseases of the Esophagus 1995 (24), the two-field lymph node dissection can be divided into three types:

* Standard 2-field: incorporates, besides a wide local excision of the primary tumor, a lymphadenectomy of the entire posterior mediastinum, from the diaphragm up to the subcarinal nodes and aortopulmonary window. In the abdomen it includes the lymph nodes along the celiac trunk, common hepatic and splenic arteries, as well as the lymph nodes along the lesser gastric curvature and in the lesser omentum (the so called D II lymphadenectomy);

* Extended 2-field: besides lymph nodes included in the standard 2-field lymphadenectomy and the right paratracheal gutter up to the nodes along the right recurrent nerve and the brachiocephalic trunk;

* Total 2-field: adding to the extended 2-field dissection, the lymph nodes located in the left paratracheal gutter including lymph nodes alongside the left recurrent nerve.

Although the high frequency of lymph node involvement above the level of the carina has been largely accepted in case of squamous cell carcinoma (25), it is less clear for adenocarcinoma. Based on the extensive work of Siewert on
1,602 adenocarcinoma cases, the risk of having lymph node involvement above the level of the carina was estimated at $5 \%$ (26). It is important to realize that more than $60 \%$ of Siewert type I adenocarcinoma underwent a transhiatal resection limiting strongly the usefulness of these results as a formal lymph node dissection above the carinal region is virtually impossible via THE.

On the contrary, in a group of adenocarcinoma of the GEJ undergoing three-hole esophagectomy with extended 2 -field lymph node dissection in all cases, the frequency of lymph node involvement in the subcarinal region or above was $23 \%$ (27). Furthermore, a study by D'Journo et al. (28) indicates clearly that in up to $25 \%$ of all patients, and in more than $35 \%$ of the $\mathrm{N}+$ patients, standard 2-field lymphadenectomy would have led to inadequate staging and, in turn, incomplete resection. This underscores the importance of lymph node dissection in defining extent of tumoral lymph node involvement which is clearly underestimated through a transhiatal approach or a standard 2-field lymphadenectomy.

Importantly, in the same study extended lymphadenectomy was found to be an independent prognosticator of improved overall and disease-free survival at multivariable analysis, suggesting a beneficial effect of the technique. The limitation of this study, however, was that in a subgroup analysis, the survival difference was only seen in the N0 patients group but not in the $\mathrm{N}+$ group, what could possibly be explained by a stage migration effect. Indeed, extended lymphadenectomy clearly decreases the proportion of 'falsenegative' patients regarding their $\mathrm{N}$-status but without necessarily increasing the overall prognosis. Conversely, standard lymphadenectomy would have resulted in a loss of chance to be cured for those patients who finally had an unknown residual disease after surgery unlikely to be treated by adjuvant therapy.

Obviously, not only the presence or absence of involved lymph nodes seems important for survival but also the number of involved lymph nodes. Indeed, in a follow up study of the earlier mentioned HIVEX trial TTE had an improved survival (64\% vs. $23 \%, \mathrm{P}=0.02)$ as compared to THE in patients with limited number of involved lymph nodes (1-8 involved lymph nodes) (16). Furthermore, in patients with low number of involved lymph nodes, anatomical location of the involvement does not seem to play such an important role in patients having undergone an extensive lymph node dissection. In a group of patients treated for mid to distal esophageal cancer by extended 2or 3-field lymphadenectomy by Watanabe et al. in Japan, 
patients presenting with a single lymph node involvement either alongside the recurrent laryngeal nerve or in the paracardial region had comparable survival (29).

\section{The three-field lymph node dissection}

The pattern of lymphatic dissemination is not restricted to the thorax and abdomen. About one in four of the patients with a distal third tumor present with metastasis in the cervical region (30). In this operation, besides the already mentioned removal of thoracic and abdominal nodes, the cervical field includes the paraesophageal nodes and the nodes lateral to the carotid vessel as well as the supraclavicular nodes. Most of the literature on three fields lymphadenectomy is coming from the far East, specifically Japan and more recently from China. Two randomized controlled prospective trials have been published $(31,32)$. However, they are somewhat out dated and were heavily criticized because of too much inherent bias issues. In a recent meta-analysis, it was clearly shown that three-field lymph node dissection can improve survival in squamous cell carcinoma (HR 0.64, 95\% CI: 0.56-0.73, $\mathrm{P}=0.000$ ), and especially for middle and proximal $1 / 3$ squamous cell carcinoma (33). For adenocarcinoma however, the advantage of adding a third field during lymph node dissection is less clear. Two Western series have shown high frequency of recurrent laryngeal nerve- or cervical lymph node involvement, even in the case of distal and GEJ adenocarcinoma with a clear impact on staging $(34,35)$. Moreover in the study by Lerut $e t$ al. (34), 13\% of patients with otherwise no evidence of nodal disease had unsuspected metastases in the cervicothoracic nodes. Based on a dismal outcome in case of cervical lymph node involvement in adenocarcinoma, with essentially no survivors beyond 2 years, the improved detection of lymph node during clinical staging based on PET or PET/CT and the increased risk of complications (anastomotic leaks, recurrent nerve palsy), the standard use of this technique has failed to be accepted for adenocarcinoma. Only patients with small number of lymph nodes involved could possibly experience improved survival after three-field lymphadenectomy $(34,35)$.

To tailor the use of three-field lymphadenectomy as to avoid unnecessary complications, one can use the possible 'sentinel' role of recurrent nerve chain lymph nodes. Indeed, in a group of patients systematically treated with three-field lymph node dissection, the incidence of cervical lymph node involvement was significantly higher in recurrent nerve-positive $(51.6 \%)$ as compared to recurrent node-negative patients $(11.6 \%)$ in patients with middle or lower esophageal cancer (36). Negative preoperative frozen section of recurrent nerve lymph nodes can preclude the need of a formal three-field lymph node dissection except for proximal esophageal cancer or in patients with clinical suspicion of lymph node involvement in the neck.

\section{Neoadjuvant treatment: change of strategy and version 2.0 of the debate}

The vast majority of trials described above mainly included patients undergoing primary surgery without neoadjuvant treatment. Nowadays, most patients with locally advanced esophageal cancer will undergo neoadjuvant treatment before surgery in particular since the publication of the CROSS trial (37). In this study, total number of resected lymph nodes was significantly lower in patients undergoing neoadjuvant treatment followed by surgery than compared with patients treated by surgery alone. Furthermore, after surgery alone, total number of resected nodes had a positive correlation with survival (HR per 10 additionally resected nodes, 0.76; $\mathrm{P}=0.007$ ), but this was no longer the case after neoadjuvant treatment (HR 1.00; $\mathrm{P}=0.98$ ), suggesting a probably relevant role of extensive lymph node dissection in patients undergoing primary surgery but not in patients undergoing surgery after neoadjuvant treatment (38).

In addition, a recent cohort study of patients who underwent esophagectomy (the majority after neoadjuvant treatment) at a high volume center in the UK has demonstrated a lack of influence of the extent of lymph node dissection on 5-year survival (39).

On the other hand, using the criteria of optimal lymph node dissection as defined by the WECC group (11) (minimum 10 lymph nodes resected for T1, 20 for T2 and 30 for T3-4 to be seen as optimal lymph node dissection), improved survival was demonstrated in case of optimal lymph node dissection as compared to suboptimal lymph node dissection in a retrospective study of patients undergoing TTE after neoadjuvant treatment (40).

Obviously, based on the existing evidence, the debate on the extent of lymph node dissection is not yet ready to subside.

\section{Complications of lymph node dissection and quality of life}

Besides the possibility that a more extensive lymph node dissection improves staging, decreases locoregional recurrences and possibly improves overall survival, 
especially in patients with limited number of involved lymph nodes, one has to take into account the risk of increased morbidity when a more extensive lymph node dissection is performed. It has been clearly shown that morbidity after extended lymphadenectomy was increased as compared to standard 2-field, especially an increased risk of respiratory complications and need of transfusion (28). The relevance of this is twofold. Firstly, there is evidence that major complications may have a negative impact on oncologic outcome in particular earlier onset of recurrence (41). Secondly, complications clearly affect negatively quality of life, an item that with the steady improvements in long-term outcome is gaining increasing attention (42). So possible advantages of more extended lymph node dissection have to be weighted keeping in mind the increased risk of complications. On the other hand, a recent nationwide, population-based cohort study in Sweden has shown that a more extensive lymph node dissection neither increased postoperative mortality (43) nor negatively influence patient's short-term or long term health-related quality of life (44) which could lead to a more liberal use of more extended lymph node dissection so as to maximize the possible improved survival brought by more extensive lymph node dissection.

\section{The Leuven point of view: a pragmatic approach}

Based on the existing evidence, it is clear that defining an evidence based surgical treatment for esophageal cancer patients is a challenge. Therefore, the Leuven group has defined a more pragmatic surgical approach to treat those.

A TTE is the mainstay of treatment for all tumors of the esophagus and GEJ as it allows probably the best chance for complete resection, optimal lymph node dissection and improved survival.

Based on the recent literature, MIE using a TTE in prone position has become our procedure of choice. In case of bulky tumor alongside the airway before or after neoadjuvant treatment, a hybrid resection using a right thoracotomy, laparoscopy and cervical anastomosis will be performed. This approach has the potential to limit complications as shown by the MIRO-trial (23) and offers to the surgeon improved control and safety when dissecting the tumor from the membranous part of the airways in order to optimize complete concologic (R0) resection and limiting the risk of perforation of the airway, which is an increased concern during thoracoscopic mobilisation of the esophagus.
An extended 2-field lymph node dissection will be performed in both approaches, with frozen section of the recurrent nerve lymph node if deemed necessary to decide whether a 3-field lymphadenectomy should be performed. In the case of a proximal tumor or in case of lymph node involvement in the cervical region (prior to neoadjuvant treatment or during surgery), a 3 -field lymph node dissection is deemed mandatory.

When minimally invasive surgery is not possible (e.g., previous thoracic or major upper GI surgery, dense fibrotic adhesions etc.), an open TTE depending on the location of the tumor (from the right or the left chest for tumors situated above or below the level of the aortic arch, respectively) and the extent of lymph node involvement to be performed.

In case of tumors invading the stomach more extensively (more than $5 \mathrm{~cm}$ along the lesser curvature), a total gastrectomy through left thoracoabdominal approach will be performed.

We reserve more tailored resection and extent of lymphadenectomy to patients with extensive co-morbidities, limited cardio-respiratory function or other particular situations.

As a result of this more patient tailored approach and being the major tertiary referral center of the country, approximately half of the patients receive a total MIE in prone position the other half being treated by the MIRO type hybrid or total open approach

\section{Acknowledgements}

None.

\section{Footnote}

Conflicts of Interest: The authors have no conflicts of interest to declare.

\section{References}

1. Clark GW, Peters JH, Ireland AP, et al. Nodal metastasis and sites of recurrence after en bloc esophagectomy for adenocarcinoma. Ann Thorac Surg 1994;58:646-53.

2. Akiyama H, Tsurumaru M, Kawamura T, et al. Principles of surgical treatment for carcinoma of the esophagus: analysis of lymph node involvement. Ann Surg 1981;194:438-46.

3. Orringer MB. Thranshiatal esophagectomy without 
thoracotomy for carcinoma of the thoracic esophagus. Ann Surg 1984;200:282-8.

4. Lerut T, Coosemans W, De Leyn P, et al. Optimizing treatment of carcinoma of the esophagus and gastroesophageal junction. Surg Oncol Clin N Am 2001;10:863-84.

5. Müller JM, Erasmi H, Stelzner M, et al. Surgical therapy of oesophageal carcinoma. Br J Surg 1990;77:845-57.

6. Altorki NK, Girardi L, Skinner DB. En bloc esophagectomy improves survival for stage III esophageal cancer. J Thorac Cardiovasc Surg 1997;114: 948-55.

7. Stein HJ, Feith M, Bruecher BL, et al. Early esophageal cancer: pattern of lymphatic spread and prognostic factors for long-term survival after surgical resection. Ann Surg 2005;242:566-73.

8. Westerterp M, Koppert LB, Buskens CJ, etal. Outcome of surgical treatment for early adenocarcinoma of the esophagus or gastro-esophageal junction. Virchows Arch 2005;446:497-504.

9. Fujita H, Sueyoshi S, Yamana H, etal. Optimum treatment strategy for superficial esophageal cancer: endoscopic mucosal resection versus radical esophagectomy. World J Surg 2001;25:424-31.

10. Nishimaki T, Suzuki T, Kanda T, et al. Extended radical esophagectomy for superficially invasive carcinoma of the esophagus. Surgery 1999;125:142-7.

11. Rizk NP, Ishwaran H, Rice TW, et al. Optimum lymphadenectomy for esophageal cancer. Ann Surg 2010;251:46-50.

12. Peyre CG, Hagen JA, DeMeester SR, etal. The number of lymph nodes removed predicts survival in esophageal cancer: an international study on the impact of extent of surgical resection. Ann Surg 2008;248:549-56.

13. Davies AR, Sandhu H, Pillai A, et al. Surgical resection strategy and the influence of radicality on outcomes in oesophageal cancer. Br J Surg 2014;101:511-17.

14. Boshier PR, Anderson O, Hanna GB. Transthoracic versus transhiatal esophagectomy for the treatment of esophagogastric cancer: a meta-analysis. Ann Surg 2011;254:894-906.

15. Hulscher JB, van Sandick JW, de Boer AG, etal. Extended transthoracic resection compared with limited transhiatal resection for adenocarcinoma of the esophagus. $\mathrm{N}$ Engl J Med 2002;347:1662-69.

16. Omloo JM, Lagarde SM, Hulscher JB, et al. Extended transthoracic resection compared with limited transhiatal resection for adenocarcinoma of the mid/distal esophagus: five-year survival of a randomized clinical trial. Ann Surg
2007;246:992-1000.

17. Connors RC, Reuben BC, Neumayer LA, et al. Comparing outcomes after transthoracic and transhiatal esophagectomy: a 5-year prospective cohort of 17,395 patients. J Am Coll Surg 2007;205:735-40.

18. Lv L, Hu W, Ren Y, et al. Minimally invasive esophagectomy versus open esophagectomy for esophageal cancer: a meta-analysis. Onco Targets Ther 2016;9:6751-62.

19. Biere SS, Cuesta MA, van der Peet DL. Minimally invasive versus open esophagectomy for cancer: a systematic review and meta-analysis. Minerva Chir 2009;64:121-33.

20. Nagpal K, Ahmed K, Vats A, et al. Is minimally invasive surgery beneficial in the management of esophageal cancer? A meta-analysis. Surg Endosc 2010;24:1621-9.

21. Biere SS, van Berge Henegouwen MI, Maas KW, etal. Minimally invasive versus open oesophagectomy for patients with oesophageal cancer: a multicentre, open-label, randomised controlled trial. Lancet 2012;379:1887-92.

22. Decker G, Coosemans W, De Leyn P, et al. Minimally invasive esophagectomy for cancer. Eur J Cardiothorac Surg 2009;35:13-20.

23. Briez N, Piessen G, Bonnetain F, et al. Open versus laparoscopically-assisted oesophagectomy for cancer: a multicentre randomised controlled phase III trial - the MIRO trial. BMC Cancer 2011;11:310.

24. Fumagalli U. Resective surgery for cancer of the thoracic esophagus. Results of a Consensus Conference held at the VIth World Congress of the International Society for Diseases of the Esophagus. Dis Esophagus 1996;9:30-8.

25. Udagawa H, Akiyama H. Surgical treatment of esophageal cancer: Tokyo experience of the three-field technique. Dis Esophagus 2001;14:110-4.

26. Feith M, Stein HJ, Siewert JR. Adenocarcinoma of the esophagogastric junction: surgical therapy based on 1602 consecutive resected patients. Surg Oncol Clin N Am 2006;15:751-64.

27. Lagarde SM, Cense HA, Hulscher JB, et al. Prospective analysis of patients with adenocarcinoma of the gastric cardia and lymph node metastasis in the proximal field of the chest. Br J Surg 2005;92:1404-8.

28. D'Journo XB, Doddoli C, Michelet P, et al. Transthoracic esophagectomy for adenocarcinoma of the oesophagus: standard versus extended two-field mediastinal lymphadenectomy? Eur J Cardiothorac Surg 2005;27:697-704.

29. Watanabe H, Kato H, Tachimori Y. Significance of extended systemic lymph node dissection for thoracic 
esophageal carcinoma in Japan. Recent Results Cancer Res 2000;155:123-33.

30. Akiyama H, Tsurumaru M, Udagawa H, et al. Radical lymph node dissection for cancer of the thoracic esophagus. Ann Surg 1994;220:364-72.

31. Kato H, Watanabe H, Tachimori Y, et al. Evaluation of neck lymph node dissection for thoracic esophageal carcinoma. Ann Thorac Surg 1991;51:931-5.

32. Nishihira T, Hirayama K, Mori S. A prospective randomized trial of extended cervical and superior mediastinal lymphadenectomy for carcinoma of the thoracic esophagus. Am J Surg 1998;175:47-51.

33. Ye T, Sun Y, Zhang Y, et al. Three-field or two-field resection for thoracic esophageal cancer: a meta-analysis. Ann Thorac Surg 2013;96:1933-41.

34. Lerut T, Nafteux P, Moons J, et al. Three-field lymphadenectomy for carcinoma of the esophagus and gastroesophageal junction in $174 \mathrm{R} 0$ resections: impact on staging, disease-free survival, and outcome: a plea for adaptation of TNM classification in upper-half esophageal carcinoma. Ann Surg 2004;240:962-72.

35. Altorki N, Kent M, Ferrara C, et al. Three-field lymph node dissection for squamous cell and adenocarcinoma of the esophagus. Ann Surg 2002;236:177-83.

36. Shiozaki H, Yano M, Tsujinaka T, et al. Lymph node metastasis along the recurrent nerve chain is an indication for cervical lymph node dissection in thoracic esophageal cancer. Dis Esophagus 2001;14:191-6.

37. van Hagen P, Hulshof MC, van Lanschot JJ, et al. Preoperative chemoradiotherapy for esophageal or junctional cancer. N Engl J Med 2012;366:2074-84.

Cite this article as: Nafteux P, Depypere L, Van Veer H, Coosemans W, Lerut T. Principles of esophageal cancer surgery, including surgical approaches and optimal node dissection (2vs. 3-field). Ann Cardiothorac Surg 2017;6(2):152-158. doi: 10.21037/acs.2017.03.04
38. Koen Talsma A, Shapiro J, Looman CW, et al. Lymph node retrieval during esophagectomy with and without neoadjuvant chemoradiotherapy: prognostic and therapeutic impact on survival. Ann Surg 2014;260:786-92

39. Lagergren J, Mattsson F, Zylstra J, et al. Extent of Lymphadenectomy and Prognosis After Esophageal Cancer Surgery. JAMA Surg 2016;151:32-9.

40. Stiles BM, Nasar A, Mirza FA, et al. Worldwide Oesophageal Cancer Collaboration guidelines for lymphadenectomy predict survival following neoadjuvant therapy. Eur J Cardiothorac Surg 2012;42:659-64.

41. Lerut T, Moons J, Coosemans W, et al. Postoperative complications after transthoracic esophagectomy for cancer of the esophagus and gastroesophageal junction are correlated with early cancer recurrence: role of systematic grading of complications using the modified Clavien classification. Ann Surg 2009;250:798-807.

42. Nafteux P, Durnez J, Moons J, et al. Assessing the relationships between health-related quality of life and postoperative length of hospital stay after oesophagectomy for cancer of the oesophagus and the gastro-oesophageal junction. Eur J Cardiothorac Surg 2013;44:525-33.

43. Lagergren J, Mattsson F, Davies A, et al. Lymphadenectomy and risk of reoperation or mortality shortly after surgery for oesophageal cancer. Sci Rep 2016;6:36092.

44. Schandl A, Johar A, Lagergren J, et al. Lymphadenectomy and health-related quality of life after oesophageal cancer surgery: a nationwide, population-based cohort study. BMJ Open 2016;6:e012624. 